\title{
Left ventricular outflow tract obstruction with systolic anterior motion of the mitral valve in patient with pericardial effusion caused by ascending aortic dissection -A case report-
}

\author{
Keun Suk Park, Hyerim Kim, Yoo Sun Jung, Hyun Joo Kim, Jung-Man Lee, Deok Man Hong, \\ Yunseok Jeon, and Jae-Hyon Bahk \\ Department of Anesthesiology and Pain Medicine, Seoul National University Hospital, Seoul, Korea
}

Left ventricular outflow tract (LVOT) obstruction with systolic anterior motion (SAM) of mitral valve is not only limited to patients with hypertrophic cardiomyopathy. A diagnosis of LVOT obstruction with SAM is important because conventional inotropic support may potentially aggravate hemodynamic deterioration. We present a case of LVOT obstruction with SAM in a patient who underwent an emergent surgery for ascending aortic dissection with pericardial effusion. The patient showed refractory hypotension after standard pharmacologic interventions during induction of anesthesia. Transesophageal echocardiography (TEE) revealed LVOT obstruction with SAM and it was managed appropriately under the guidance of TEE. Intraoperative TEE can play an important role in diagnosis and management of LVOT obstruction with SAM caused by pericardial effusion. (Korean J Anesthesiol 2013; 64: 73-76)

Key Words: Ascending aortic dissection, Hypotension, LVOT obstruction, Pericardial effusion, Systolic anterior motion of mitral valve.

Left ventricular outflow tract (LVOT) obstruction with systolic anterior motion (SAM) of mitral valve is commonly encountered in patients with hypertrophic cardiomyopathy [1], but it can also be observed in other conditions [2-7]. Dynamic interaction between anatomy and mechanics of the heart may cause LVOT obstruction with SAM which can potentially deteriorate the hemodynamics of the circulatory system.

We present a case of LVOT obstruction with SAM in a patient who had a large amount of pericardial effusion which resulted from ascending aortic dissection. Pericardial effusion may restrict left ventricular volume and therefore result in hyperdynamic left ventricle which may predispose to LVOT

Received: February 3, 2012. Revised: March 4, 2012. Accepted: March 5, 2012.

Corresponding author: Jae-Hyon Bahk, M.D., Ph.D., Department of Anesthesiology and Pain Medicine, Seoul National University Hospital, Seoul National University College of Medicine, 101, Daehak-ro, Jongno-gu, Seoul 110-744, Korea. Tel: 82-2-2072-2469, Fax: 82-2-747-5639, E-mail: bahkjh@snu.ac.kr

C) This is an open-access article distributed under the terms of the Creative Commons Attribution Non-Commercial License (http:// creativecommons.org/licenses/by-nc/3.0/), which permits unrestricted non-commercial use, distribution, and reproduction in any medium, provided the original work is properly cited. 
obstruction with SAM. Hypotensive agents used for the control of blood pressure in aortic dissection can potentially reduce preload and vascular resistance of the circulatory system, which may aggravate LVOT obstruction with SAM. The use of Intraoperative transesophageal echocardiography is important to recognize this pathophysiology in clinically unstable patients, and aids to establish an appropriate therapeutic strategy.

\section{Case Report}

A 72-year-old woman ( $145 \mathrm{~cm}, 47 \mathrm{~kg}$ ) who was diagnosed with ascending aortic dissection presented for ascending aorta and hemiarch replacement surgery. She visited the emergency department with chest discomfort, nausea, and vomiting which started the day before. Her past medical history included common bile duct stone and hypertension that was controlled with an angiotensin receptor blocker. On arrival, her vital signs were stable with a blood pressure of 147/100 $\mathrm{mmHg}$ and heart rate of 86 beats per minute (bpm). Physical examination revealed abdominal tenderness on her epigastric area. Her chest X-ray showed bilateral pleural effusion, while her electrocardiogram (ECG) was normal . A chest computed tomography (CT) revealed Stanford type A aortic dissection that started from the aortic root through the proximal descending aorta that resulted in a large amount of pericardial effusion. Immediate emergent operation was planned, and transthoracic echocardiography (TTE) could not be performed.

When she arrived in the operating room, electrocardiography, pulse oxymetry and invasive blood pressure monitoring was conducted. Her blood pressure was managed with continuous infusion of nicardipine $2 \mu \mathrm{g} / \mathrm{kg} / \mathrm{min}$, esmolol 100 $\mu \mathrm{g} / \mathrm{kg} / \mathrm{min}$, nitroglycerin $2 \mu \mathrm{g} / \mathrm{kg} / \mathrm{min}$, and nitroprusside 0.5 $\mu \mathrm{g} / \mathrm{kg} / \mathrm{min}$. The initial vital signs checked in the operating room were stable with blood pressure of 100/56 $\mathrm{mmHg}$ and heart rate of $86 \mathrm{bpm}$. Induction of anesthesia was performed with $7 \mathrm{mg}$ of midazolam, $50 \mu \mathrm{g}$ of sufentanil, and $10 \mathrm{mg}$ of vecuronium. After the injection of the induction agents, her blood pressure decreased to $76 / 46 \mathrm{mmHg}$, and the maximum heart rate noted at the time was $100 \mathrm{bpm}$. The infusion of the hypotensive agents (nicardipine, esmolol, nitroglycerin and nitroprusside) was discontinued, and subsequently $10 \mathrm{mg}$ of ephedrine, 30 $\mu \mathrm{g}$ of phenylephrine and rapid volume infusion using lactate Ringer's solution were administered. In response to persistent hypotension, $10 \mathrm{mg}$ of ephedrine was administered again, but hypotension was exacerbated resulting in a blood pressure of 66/42 mmHg. After endotracheal intubation, transesophageal echocardiography (TEE) was performed to evaluate cardiac function and to guide the hemodynamic treatment. TEE showed a large amount of pericardial effusion (Fig. 1), septal hypertrophy, severe eccentric mitral regurgitation and systolic anterior motion (SAM) of mitral valve combined with dynamic left ventricular outflow tract (LVOT) obstruction (Fig. 2). To improve LVOT obstruction with SAM, $50 \mathrm{mg}$ of esmolol and several $100 \mu \mathrm{g}$ boluses of phenylephrine, and more rapid volume resuscitation (300 $\mathrm{ml}$ of lactate Ringer's solution and $300 \mathrm{ml}$ of hydroxyethyl starch solution) were administered.

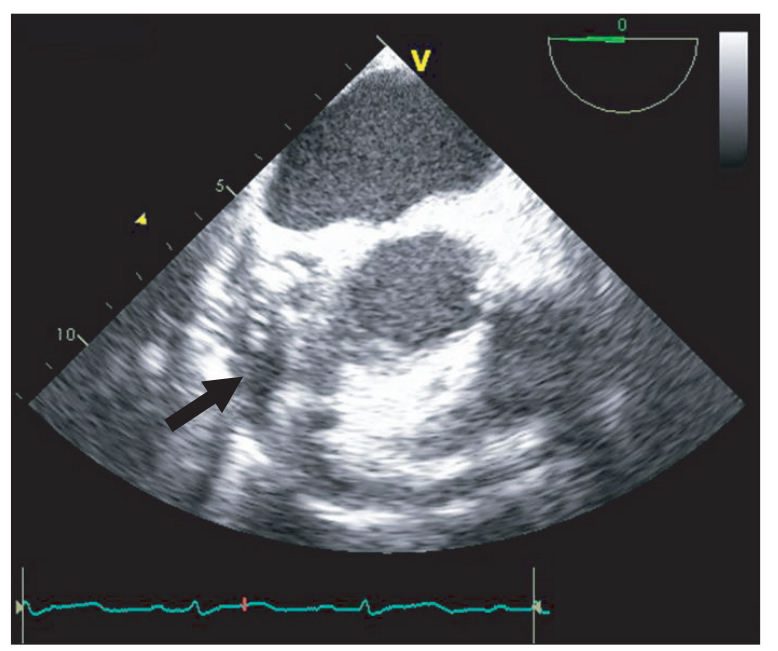

Fig. 1. Transesophageal echocardiography showing a large amount of pericardial effusion (arrow).
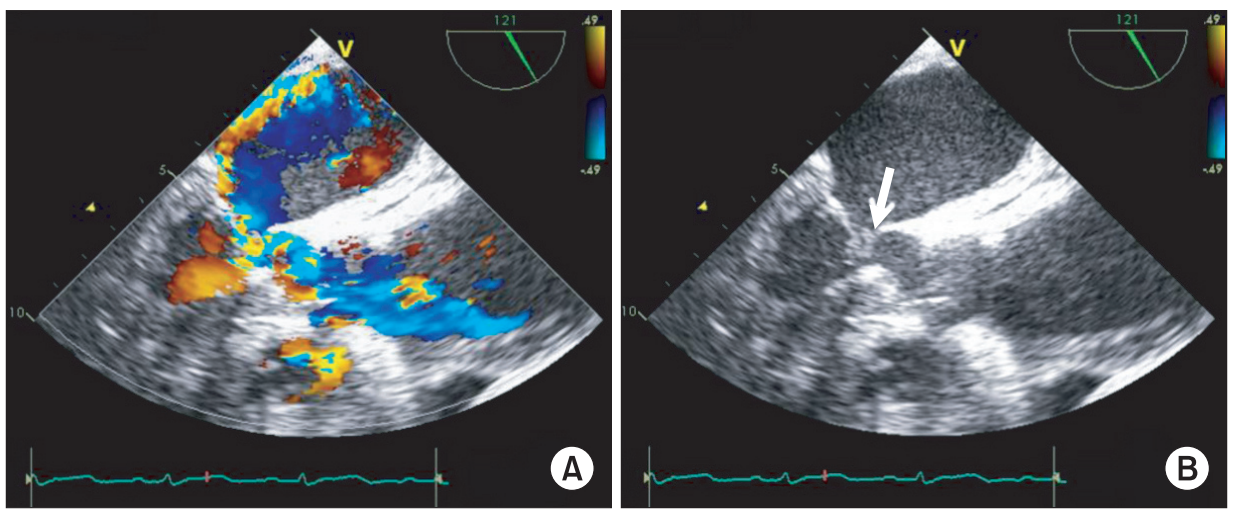

Fig. 2. (A) Color Doppler image showing mitral regurgitation and flow acceleration in the left ventricular outflow tract. (B) Without color Doppler, the transesophageal echocardiography showing septal hypertrophy and left ventricular outflow obstruction with systolic anterior motion of mitral valve (arrow). 
Afterwards, the patient's blood pressure became stable at 102/56 mmHg and heart rate of $88 \mathrm{bpm}$. LVOT obstruction with SAM improved, and mitral regurgitation decreased to a mild level. A triple lumen catheter with introducer (Advanced Venous Access ${ }^{\circledR}$ ) was placed in the right internal jugular vein and a pulmonary artery catheter was inserted to monitor the central venous and pulmonary artery pressure. Anesthesia was maintained with propofol $(1.5-2.5 \mu \mathrm{g} / \mathrm{ml})$, and remifentanil $(8-20 \mathrm{ng} / \mathrm{ml})$ by target controlled infusion system under bispectral index monitoring. Vecuronium was infused continuously ( $1 \mu \mathrm{g} / \mathrm{kg} / \mathrm{min})$ for muscle relaxation.

Following sternotomy and opening of pericardium, about 200 $\mathrm{ml}$ of bloody pericardial effusion was drained for evacuation. After the drainage, residual SAM and mitral regurgitation completely disappeared on TEE. An ascending aorta and hemiarch replacement was conducted under cardiopulmonary bypass (CPB). While attempting to wean from CPB, SAM with LVOT obstruction reappeared. Esmolol was administered with bolus (50 mg) and continuous infusion ( $50 \mu \mathrm{g} / \mathrm{kg} / \mathrm{min})$. Afterwards, SAM with LVOT obstruction improved, and patient was weaned from CPB successfully. Postoperative TTE was performed one week after surgery and revealed no evidence of SAM. She was discharged from the hospital 11 days following the operation without major complications.

\section{Discussion}

Hypertrophic cardiomyopathy is the most common cause of dynamic left ventricle outflow tract (LVOT) obstruction with systolic anterior motion (SAM) of mitral valve [1]. However, LVOT obstruction also occurs in other conditions without cardiac history, such as mitral valve repair [2], myocardial infarction [3], dobutamine stress test [4], liver transplantation [5,6] and catecholamine infusion [7]. LVOT obstruction with SAM is induced by structural abnormalities such as small hyperdynamic left ventricular cavity, narrow LVOT, sigmoid septum, and displaced mobile elongated mitral leaflets [8]. This mechanism may be dynamic depending on the hemodynamics and anatomic conditions.

In this case, LVOT obstruction with SAM was found during induction of anesthesia and disappeared after the drainage of pericardial effusion. Although it is uncertain exactly when LVOT obstruction with SAM developed, this patient showed hemodynamic deterioration after the administration of anesthetic agents. Several factors might have been involved which led to the development of this condition. First, this patient had septal hypertrophy and small ventricular volume restricted by pericardial effusion which might have predisposed to LVOT obstruction. Second, preoperative use of hypotensive agents would have decreased preload and systemic vascular resistance (SVR), and anesthetic agents might have caused further reduction of SVR. Third, hyperdynamic activity of the left ventricle to compensate pericardial effusion might have aggravated LVOT obstruction with SAM, as evidenced by disappearance of SAM after the drainage of bloody pericardial effusion. This decrease of preload, combined with the sudden reduction of SVR, may lead LVOT obstruction with SAM in patients with predisposing factors.

Patients with aortic dissection are usually managed with hypotensive agents to control hypertension which may exacerbate the dissection. Sometimes pericardial effusion or even cardiac tamponade could be accompanied with aortic dissection. These pre-existing conditions may elevate the risk of LVOT obstruction due to decreased SVR and hyperdynamic cardiac status. In cases of relative hypovolemia, hyperdynamic cardiac state is further worsened and LVOT obstruction may occur.

Treatment of LVOT obstruction with SAM consists of increasing preload and decreasing the hyperdynamic state of the heart. Preload can be increased by aggressive intravascular volume infusion to increase the left ventricular end diastolic dimension. Increasing of SVR using vasopressors can be helpful by decreasing the Venturi effect on the LVOT. Beta adrenergic receptor blockers have proven effective in the treatment of hypertrophic cardiomyopathy, and their efficacy is also valid in the management of LVOT obstruction with SAM [9]. In the current case, massive volume infusion was performed and intravenous esmolol and phenylephrine was administered. Positive inotropes may aggravate LVOT obstruction with SAM and induce paradoxic decrease in blood pressure. Inotropic support and volume resuscitation are preferentially considered in cases of hypotension during anesthesia induction; however, inotropic agents are known to induce the hyperdynamic state and worsen mitral regurgitation with SAM in patients with predisposing LVOT obstruction. On the other hand, the treatment strategy for LVOT obstruction with SAM may aggravate hemodynamic deterioration if hypotension was not caused by SAM. Therefore, a differential diagnosis using echocardiography is essential for refractory hypotension.

LVOT obstruction with SAM is suspected with hemodynamic deterioration after catecholamine infusion, and new development of systolic murmur. However, definitive diagnosis requires performing echocardiography. In this case, TEE played an essential role in the diagnosis and management of LVOT obstruction with SAM. Findings on TEE revealed that administration of phenylephrine and esmolol and infusion of a large amount of fluid led to hemodynamic improvement. Although LVOT obstruction with SAM is diagnosed with echocardiography, CT scan or magnetic resonance imaging (MRI) is preferred in cases of aortic dissection because of their ability to evaluate the anatomical structures and the 
extent of the disease. However, CT scan and MRI are not suitable in evaluating functional and anatomic status of the aortic valve and they do not evaluate the dynamicity of left ventricular dysfunction [10]. In the current case, the dynamic effects of pericardial effusion and septal hypertrophy which may predispose to LVOT obstruction were discovered in the operating room because preoperative echocardiography was not performed.

In conclusion, LVOTO with SAM should be considered as a cause of refractory hypotension in patients with large amounts of pericardial effusion. Echocardiography is essential for the diagnosis and exact treatment. In cases of refractory hypotension which do not respond to conventional inotropic support, TEE should be performed to evaluate dynamic cardiac function.

\section{References}

1. Maron MS, Olivotto I, Betocchi S, Casey SA, Lesser JR, Losi MA, et al. Effect of left ventricular outflow tract obstruction on clinical outcome in hypertrophic cardiomyopathy. N Engl J Med 2003; 348: 295-303.

2. Glas KE, Shanewise JS, Guyton RA. An unusual cause of left ventricular outflow tract obstruction after mitral valve repair. Anesth Analg 2004; 99: 38-40.

3. Chockalingam A, Tejwani L, Aggarwal K, Dellsperger KC. Dynamic left ventricular outflow tract obstruction in acute myocardial infarction with shock: cause, effect, and coincidence. Circulation 2007; 116: e110-3.

4. Roldán FJ, Vargas-Barrón J, Espinola-Zavaleta N, Keirns C, RomeroCárdenas A. Severe dynamic obstruction of the left ventricular outflow tract induced by dobutamine. Echocardiography 2000; 17: 37-40.

5. Aniskevich S, Shine TS, Feinglass NG, Stapelfeldt WH. Dynamic left ventricular outflow tract obstruction during liver transplantation: the role of transesophageal echocardiography. J Cardiothorac Vasc Anesth 2007; 21: 577-80.

6. Lee AR, Kim YR, Ham JS, Lee SM, Kim GS. Dynamic left ventricular outflow tract obstruction in living donor liver transplantation recipients -A report of two cases. Korean J Anesthesiol 2010; 59 Suppl: S128-32.

7. Mingo S, Benedicto A, Jimenez MC, Pérez MA, Montero M. Dynamic left ventricular outflow tract obstruction secondary to catecholamine excess in a normal ventricle. Int J Cardiol 2006; 112: 393-6.

8. Levine RA, Vlahakes GJ, Lefebvre X, Guerrero JL, Cape EG, Yoganathan AP, et al. Papillary muscle displacement causes systolic anterior motion of the mitral valve. Experimental validation and insights into the mechanism of subaortic obstruction. Circulation 1995; 91: 1189-95.

9. Maron BJ. Hypertrophic cardiomyopathy: a systematic review. JAMA 2002; 287: 1308-20.

10. Booher AM, Eagle KA. Diagnosis and management issues in thoracic aortic aneurysm. Am Heart J 2011; 162: 38-46. 